\title{
Transatlantica
}

Revue d'études américaines. American Studies Journal

\section{Richard Anker, Henry James, Le principe spectral de la représentation, Paris, Hermann, 2012, 460 pages}

\section{Michel Imbert}

\section{(2) OpenEdition}

1 Journals

\section{Édition électronique}

URL : https://journals.openedition.org/transatlantica/6057

DOI : $10.4000 /$ transatlantica.6057

ISSN : 1765-2766

Éditeur

Association française d'Etudes Américaines (AFEA)

\section{Référence électronique}

Michel Imbert, «Richard Anker, Henry James, Le principe spectral de la représentation, Paris, Hermann, 2012, 460 pages », Transatlantica [En ligne], 2 | 2012, mis en ligne le 22 mai 2013, consulté le 06 avril 2023. URL : http://journals.openedition.org/transatlantica/6057 ; DOI : https://doi.org/10.4000/ transatlantica.6057

Ce document a été généré automatiquement le 6 avril 2023.

Creative Commons - Attribution - Pas d'Utilisation Commerciale - Pas de Modification 4.0 International - CC BY-NC-ND 4.0

https://creativecommons.org/licenses/by-nc-nd/4.0/ 


\title{
Richard Anker, Henry James, Le principe spectral de la représentation, Paris, Hermann, 2012, 460 pages
}

\author{
Michel Imbert
}

1 Le titre de l'ouvrage de Richard Anker, Henry James, Le principe spectral de la représentation est emprunté à une citation d'Henry James («the very presence of the haunting principle, as it were, of my thought ", 10). Il met l'accent sur le phénomène de la hantise et son rôle déterminant dans la genèse des représentations artistiques. À partir d'un corpus restreint d'œuvres publiées entre 1890 (The Tragic Muse) et 1908 ("The Jolly Corner »), comprenant entre autres The Turn of the Screw (1898), Richard Anker s'efforce de mettre au jour une scène « inaugurale » (23) plutôt que " primitive » (173), qui ne manque pas de se reproduire et qui serait la matrice invariante des fictions d'Henry James, indéfiniment répétée de récit en récit. Il met en lumière l'épreuve primordiale du trouble et de l'effroi qui hante l'esprit des personnages qui y sont exposés et les conduit à tenter de se ressaisir par l'artifice controuvé de l'imitation, pratiquée diversement selon leurs talents artistiques personnels (théâtre, peinture, roman). Les récits retracent les circonvolutions d'une conscience désemparée qui s'ingénie néanmoins à revenir progressivement à elle-même, mais qui ne parvient jamais à se représenter parfaitement l'obsédant objet de ses pensées car elle n'est jamais, à proprement parler, en pleine possession de ses moyens d'expression. À l'instar du comédien de Diderot, le personnage de James, n'étant rien, doit, « mime de rien » (212) selon l'expression de Philippe Lacoue-Labarthe, se représenter mentalement le théâtre du monde pour se constituer en tant que sujet et tenter de parer à sa vacance fondamentale. Son don de mimétisme dérive de l'expérience marquante d'une dissonance intime, jamais totalement surmontée. C'est ce processus complexe de défaillance et reconstitution de soi par l'intermédiaire de ses représentations mentales qui est décrit au moyen de la formule lapidaire de "déconstruction mimétique " $(177,212)$. C'est ainsi que Richard Anker décortique la conscience de soi au fil des romans, qu'il décrit les tours et les détours d'un esprit 
spéculatif dont l'image stylisée qu'il élabore en vient, invariablement, à être altérée par l'écho discordant d'une altérité irréductible qu'il ne parvient pas à se figurer.

2 Le premier chapitre retrace ainsi la naissance d'une vocation artistique dans The Tragic Muse. Richard Anker analyse la relation de "rivalité mimétique » qui implicitement unit et oppose dans le roman le peintre, la comédienne et le narrateur, figure du romancier; le propre des «sister arts» et des sens auxquels ils s'adressent prioritairement est ainsi de prendre la relève les uns des autres, selon des degrés de spiritualisation croissants, au fur et à mesure que l'esprit, paradoxalement, œuvre à s'objectiver. Une hiérarchie des arts, un système des beaux-arts considérés comme autant de "suppléments ", autrement dit, de formes vicariantes, s'esquisse, depuis l'art dramatique jusqu'à l'art du récit en passant par la représentation picturale, envisagés comme autant de modalités par procuration de cette méthode scénique («scenic method») chère à James (372), autrement dit, autant de manières imparfaites de se mettre en scène, de sublimer une partition antérieure dans le théâtre intérieur de la conscience. La première partie consacrée exclusivement à The Tragic Muse qui porte sur la correspondance entre les arts et sur la préhistoire prophétique de la conscience tragique est une contribution majeure à une œuvre relativement moins étudiée que d'autres grands romans. Richard Anker souligne à quel point le rôle annonciateur de Gabriel Nash a été minimisé au profit de Myriam Rooth et Nick Dormer, même par des critiques aussi perspicaces que John Carlos Rowe ou Sara Blair (138).

Les second volet de cette étude ( l'intrigue du moi ») met en lumière le retour de l'irreprésentable sur la scène hantée de la conscience à travers l'étude de deux nouvelles, «The Jolly Corner » et "The Beast in the Jungle». De fait, il est un reste inassimilable, réfractaire à cette économie solipsiste, qui en dépit des transferts et des conversions d'art en art, s'affaire à conjurer indéfiniment le spectre de sa propre défaillance intime. Les histoires de revenants, l'autre versant de l'œuvre de James qui se détourne du roman réaliste, exhument ce résidu innommable qui contrarie les spéculations spéculaires d'une conscience dramatiquement solitaire. Le second chapitre décrit ces expériences de rechute et d'effarement, de "bewilderment », pour reprendre un terme qu'affectionnait James, s'accompagnant d'un trouble du langage qui reconduit le sujet à l'épreuve première de l'effondrement qui est son fondement occulte. Le symptôme en est la perte de l'usage de la parole ou la régression à un état antérieur préverbal. Il est donné à Spencer Brydon dans "The Jolly Corner" d'appréhender l'étrangeté de l'autre en lui, en prêtant attention à l'écho de sa propre voix. Il y a dans cette seconde partie de belles pages sur l'exposition à un arrière-fond confus de bruits et de sons, à un champ de forces qui déjà défigurent ou qui ne sont pas encore des formes réfléchies. Ainsi réapparaît tout le spectre du matériau verbal mêlé de sons impurs, escamoté par une prétendue maîtrise de la langue. Les récits d'Henry James se mettraient en abyme en représentant le principe même de leur économie, le phénomène de la hantise, «the haunting principle ", son " principe spectral » car, de retournement en retournement (selon la belle formule d'Évelyne Labbé, citée page 167, bien que l'auteur s'en démarque (193-4), l'étude reconduit à ce motif obsédant que l'auteur nomme «mimésis originaire » ou "mimésis généralisée »: «En effet, tout commence chez James à partir d'un retour mimétique qui suspend l'activité représentante, fictionnante du sujet (si tant est que cette activité lui soit propre) tout en le constituant ». Le récit dont la finalité est cathartique retrace le cycle inéluctable d'un affolement, inaugural et final, qui touche à la maitrise de la langue : « Horror, with 
the sight, had leaped into his throat, gasping there in a sound he couldn't utter » (203). L'horreur contenue n'en finit pas de recommencer à surgir comme un grand fauve qui vous prend à la gorge. L'esprit hanté par une voix intérieure se coupe et, en proie à une peur panique, en vient à ravaler ses mots.

La troisième partie, intitulée "l'impropriété du propre » porte sur "A London Life », « The Turn of the Screw » et "The Visits ». Richard Anker s'attache à mettre en lumière au cœur du récit le retour à l'expérience originaire de la "dépropriation ", marquée par le brouillage des oppositions entre le réel et le fictif, le familier et l'étrange, la vie et la mort $(262,392)$. On appréciera en particulier les observations au sujet des deux orphelins, depuis toujours "dépossédés " (" dispossessed ») et dont le pouvoir d'imitation est infini pour cette raison même: "Miles (My-less) et la petite Flora (dont le nom renvoie à ces fleurs de rhétorique que l'on appelle des tropes) possèdent, par leur privation même d'identité propre, des 'facilités pour tout', une 'faculté générale', observe la gouvernante, qui n'est autre que la faculté mimétique » (393). Résumant son propos, Richard Anker énonce la double contrainte ou la double allégeance qui sous-tend l'œuvre de James: «Assujetti à une exigence de propriété, d'un côté, et astreint à une revenance symbolique dépropriante de l'autre, ne serait-ce que parce que cette dernière est la condition radicale de tout venir à soi, le sujet comparaît devant, sans s'harmoniser avec, sa condition mimétique lors du suspens. Le suspens accuse toujours l'incongruité des allégeances, et ainsi déconstruit d'une certaine manière l'hégémonie du propre » (401). Cette étude qui emprunte certains de ces concepts à Philippe Lacoue-Labarthe, à Jacques Derrida, à Jean-Luc Nancy ainsi qu'à Husserl, à Levinas et à Maurice Blanchot, tout en se montrant constamment attentive au détail de l'œuvre de James en contrepoint et aux résonances subtiles de motifs de récit en récit, cerne de façon très personnelle, avec une grande rigueur, l'émergence de la subjectivité, la naissance du sujet sur fond de disjonction, qui serait le point aveugle des représentations. Le sujet se constitue en posant devant lui, comme en miroir, une figure suppléant à son propre défaut originaire, mais «loin de fonder le sujet, le langage le bannit intimement de lui-même, le rapporte à son étrangeté irréductible » (195) ; « Des paroles jaillissent sur scène, on ne sait pas d'où » (230).

5 Entre autres citations que Richard Anker a su débusquer dans une œuvre foisonnante, en voici une qui pourrait résumer schématiquement son propos, en guise de conclusion. Le credo artistique de James pourrait être résumé par ce qu'il écrit, en 1865 déjà, dans un essai sur Whitman: «You must be possessed, and you must strive to possess your possession » (Literary Criticism, I, 634, cité 102-3).

INDEX

Thèmes : Recensions 
AUTEUR

MICHEL IMBERT

Université Paris-Diderot 